\title{
Estrutura e dinâmica comparativa entre cerradão distrófico e mesotrófico
}

\author{
Gabriel Venâncio Pereira Mariano ${ }^{1 *}$, Vanuza Pereira Garcia da Silva ${ }^{1}$, Lilian Cristina da Silva Santos ${ }^{1}$, \\ Winy Kelly Lima Pires ${ }^{1}$, Murillo de Carvalho Araújo do Carmo ${ }^{1}$, Vagner Santiago do Vale ${ }^{1}$
}

\begin{abstract}
RESUMO: Os solos do Cerrado se caracterizam pela alta intemperização e lixiviação, com baixas concentrações de nutrientes e índices elevados de alumínio. Com isso a vegetação lenhosa no cerradão sofre com déficits hídricos e nutricionais, diminuindo dinâmicas vegetacionais. O objetivo deste estudo foi comparar a sucessão ecológica no cerradão, analisada em dois grandes grupos de solos: distrófico e mesotrófico, a fim de aferir em qual destes, esta sucessão ocorria de maneira mais acelerada. Foi realizado a amostragem de vegetação em duas áreas, cerradão distrófico (CD) e cerradão mesotrófico (CM), através do monitoramento durante 5 anos, levando em consideração os indivíduos vivos e com circunferência a altura do peito superior ou igual a $15 \mathrm{~cm}$. As mudanças em escala populacional foram calculadas através das taxas de dinâmicas anuais, como mortalidade, decremento, recrutamento e incremento. Foram coletados dados de 88 espécies na primeira vistoria e 87 na segunda para CM e 84 e 85 para CD, na primeira e segunda vistoria respectivamente. O número de indivíduos passou de 1345 para 1407 no CM e 1796 no tempo inicial (T0) para 1683 no tempo final (T5) no CD. No CM a área basal foi de $18.128 \mathrm{~m}^{2}$ e $19.661 \mathrm{~m}^{2}$ e de $18.243 \mathrm{~m}^{2}$ para $18.953 \mathrm{~m}^{2}$ no CD. Com relação as taxas de dinâmica entre CD e CM, não ocorreram grandes divergências, e as taxas de recrutamento das espécies principais foram superiores ás taxas de mortalidade, semelhante ao incremento em relação ao decremento, o que demonstra uma maior estabilidade ambiental na região de CM. As espécies do cerrado distrófico apresentaram taxas de dinâmica mais estáveis enquanto no cerradão mesotrófico apresentaram maior variação entre as espécies principais.
\end{abstract}

Palavras-chave: taxas de dinâmica, monitoramento, fertilidade do solo.

\section{Structure and comparative dynamics between dystrophic and mesotrophic cerradão}

\begin{abstract}
Cerrado soils are characterized by high weathering and leaching, with low concentrations of nutrients and high levels of aluminum. With this the woody vegetation in the cerradão suffers with water and nutritional deficits, diminishing vegetative dynamics. The objective of this study was to compare the ecological succession in the cerradão, analyzed in two large groups of soils: dystrophic and mesotrophic, in order to ascertain in which of these, this succession occurred in a more accelerated way. Vegetation sampling was carried out in two areas, dystrophic cerradão (CD) and mesotrophic cerradão (CM), trough monitoring for 5 years, taking alive individuals and having a chest height of $15 \mathrm{~cm}$ or greater. Changes in population scale were calculated through annual dynamics rates, such as mortality, decrement, recruitment, and increase. Data were collected from 88 species in the first survey and 87 in the second to $\mathrm{CM}$ and 84 and 85 to $\mathrm{CD}$ in the first and second survey respectively. The number of individuals increased from 1345 to 1407 in CM and 1796 in the initial time (T0) to 1683 in the final time (T5) in the CD. In CM the basal area was 18,128 $\mathrm{m}$ and $19,661 \mathrm{~m}^{2}$ and from $18,243 \mathrm{~m}^{2}$ to $18,953 \mathrm{~m}^{2}$ on the CD. Regarding the dynamics rates between CD and CM, there were no major divergences, and the recruitment rates of the main species were higher than the mortality rates, similar to the increase in relation to the decrease, which demonstrates a greater environmental stability in the CM region. The species of the dystrophic cerradão had more stable dynamics rates while in the mesotrophic cerradão they presented greater variation among the main species.

Keywords: dynamic rates, monitoring, soil fertility.
\end{abstract}

\section{INTRODUÇÃO}

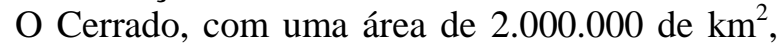
corresponde a um conjunto de ecossistemas de savanas, matas e campos, distribuídos em diferentes tipos fitofisionômicos, localizados em sua maioria, no Brasil Central (KLINK; MACHADO, 2005). Os solos do Cerrado se caracterizam pela alta

intemperização e lixiviação, com baixas concentrações de nutrientes e índices elevados de alumínio (SOUSA; LOBATO, 2004). Estas características podem ser atribuídas a textura do solo, principal parâmetro que determina suas qualidades (SANTOS et al, 2008), sendo de 
fundamental importância na retenção de umidade, capacidade de drenagem e disponibilidade de nutrientes no solo (WALTER, 2006).

Uma das principais questões sobre o bioma do Cerrado é a determinação dos fatores responsáveis pela sua distribuição e pela dinâmica das suas fitofisionomias (HENRIQUES, 2005), tais como drenagem, presença de lençol freático e fertilidade (HARIDASAN, 1992). O cerradão é um tipo fitofisionômico de formações florestais, presente em $1 \%$ do Bioma (MARIMON JUNIOR; HARIDASAN, 2005), que ocorre em solos distróficos ou mesotróficos (HARIDASAN, 1992), com composição florística variável. A ocorrência do cerradão está relacionada a um gradiente de fertilidade dos solos, podendo ocorrer em solos muito ou pouco férteis (Lopes \& Cox 1992).

A vegetação lenhosa no cerradão sofre com déficits hídricos e nutricionais acentuados, devido a maior densidade de árvores com sistemas radiculares profundos, ocorrendo uma sobreposição dessas raízes e, consequentemente, competição dos microorganismos pelos recursos, devido ao esgotamento das reservas do subsolo (NAVESBARBIERO et al., 2000). O objetivo deste estudo foi comparar a sucessão ecológica no cerradão, analisada em dois grandes grupos de solos: distrófico e mesotrófico, a fim de aferir em qual destes, esta sucessão ocorria de maneira mais acelerada.

\section{MATERIAL E MÉTODOS}

O presente estudo foi realizado em duas áreas, cerradão distrófico $(\mathrm{CD})$ e cerradão mesotrófico (CM), formações florestais do bioma Cerrado com características distintas, classificadas em relação a fertilidade do solo.

Para amostragem da vegetação foi realizado o monitoramento durante 5 anos nos dois cerradões, levando em consideração os indivíduos vivos e com circunferência à altura do peito $(1,30 \mathrm{~m})$ superior ou igual a $15 \mathrm{~cm}(\mathrm{CAP} \geq 15 \mathrm{~cm})$ foram amostrados, na presença de bifurcações todas aferidas o CAP.

As mudanças em escala populacional foram calculadas através das taxas de dinâmicas anuais: mortalidade (M) (Equação 1), decremento (P) (Equação 2), recrutamento (R) (Equação 3) e incremento $(\mathrm{G})$ (Equação 4) por meio das expressões exponenciais descritas em Sheil et al., (1995, 2000):

$$
\begin{aligned}
& M=\left\{1-\left[\frac{(N 0-m)}{N 0}\right]^{\frac{1}{t}}\right\} x 100 \\
& P=\left\{1-\left[\frac{(A B 0-A B m+A B d)}{A B 0}\right]^{\frac{1}{t}}\right\} \times 100 \text { (Equação 2) } \\
& R=\left[1-\left(\frac{1-r}{N t}\right)\right] \times 100 \\
& G=\left\{1-\left[\frac{(A B r+A B g)}{A B t}\right]^{\frac{1}{t}}\right\} \times 100 \quad \text { (Equação 4) }
\end{aligned}
$$

em que: $t$ é o tempo decorrido entre os inventários; $N O$ e $N t$ são, respectivamente, o número inicial e final de indivíduos arbóreos; $m$ e $r$ são, respectivamente, o número de árvores mortas $\mathrm{e}$ recrutas; $A B O$ e $A B t$ são, respectivamente, as áreas basais inicial e final das árvores; $A B m$ é a área basal das árvores mortas; $A B r$ é a área basal dos recrutas; $A B d$ e $A B g$ são, respectivamente, o decremento (por meio de quebra ou perda parcial do tronco) e o incremento em área basal das árvores sobreviventes.

\section{RESULTADOS E DISCUSSÕES}

A partir dos levantamentos realizados nas duas áreas, foram coletados dados de 88 espécies no cerradão mesotrófico $(\mathrm{CM})$ na primeira vistoria e 87 na segunda, e 84 espécies na área de cerradão

\begin{tabular}{|c|c|c|c|c|c|c|c|c|c|}
\hline \multirow[b]{3}{*}{ Espécies } & \multicolumn{4}{|c|}{ Mesotrófico } & \multirow[b]{3}{*}{ Espécies } & \multicolumn{4}{|c|}{ Distrófico } \\
\hline & \multicolumn{2}{|c|}{ Densidade } & \multicolumn{2}{|c|}{ Área Basal $\left(\mathrm{m}^{2}\right)$} & & \multicolumn{2}{|c|}{ Densidade } & \multicolumn{2}{|c|}{ Área Basal $\left(\mathbf{m}^{2}\right)$} \\
\hline & T0 & T5 & T0 & T5 & & T0 & T5 & T0 & T5 \\
\hline Myracrodruon urundeuva & 125 & 135 & 11349,6 & 13047,7 & Tachigali vulgaris & 321 & 350 & 42328,0 & 55963,4 \\
\hline Terminalia argentea & 124 & 124 & 31900,8 & 34099,1 & Qualea grandiflora & 193 & 167 & 44794,8 & 39732,2 \\
\hline Qualea grandiflora & 108 & 108 & 15680,3 & 16453,5 & Miconia albicans & 181 & 22 & 7552,7 & 1048,5 \\
\hline Magonia pubescens & 105 & 104 & 10902,3 & 10995,5 & Xylopia aromatica & 126 & 109 & 4442,1 & 4450,8 \\
\hline Roupala montana & 79 & 77 & 8466,9 & 9245,7 & $\begin{array}{l}\text { Virola sebifera } \\
\text { Cardiopetalum }\end{array}$ & 121 & 169 & 4306,3 & 7195,6 \\
\hline Callisthene fasciculata & 75 & 81 & 14282,4 & 16342,1 & calophyllum & 101 & 123 & 2734,9 & 3575,8 \\
\hline Dilodendron bipinnatum & 63 & 65 & 13158,0 & 14382,0 & Casearia grandiflora & 84 & 116 & 3463,5 & 5426,3 \\
\hline Myrsine umbellata & 59 & 73 & 5267,5 & 7135,2 & Siparuna guianensis & 78 & 78 & 2329,8 & 2330,1 \\
\hline Lafoensia pacari & 50 & 43 & 6900,0 & 6927,9 & Tapirira guianensis & 60 & 66 & 6384,7 & 7300,1 \\
\hline Guettarda scabra & 41 & 42 & 2210,2 & 2405,6 & Myrsine lancifolia & 53 & 25 & 1734,1 & 1009,2 \\
\hline Rhamnidium elaeocarpum & 39 & 53 & 3371,1 & 4356,4 & Terminalia brasiliensis & 50 & 50 & 11665,6 & 12274,1 \\
\hline
\end{tabular}
distrófico (CD) na primeira amostragem e 85 na segunda (Tabela 1).

Tabela 1 - Densidade populacional de espécies amostradas em dois tempos de medição no cerradão distrófico (CD) e cerradão mesotrófico $(\mathrm{CM})$ com suas respectivas áreas basais $\left(\mathrm{m}^{2}\right)$. T0 = tempo inicial; T5 = tempo final de medição. 


\begin{tabular}{|c|c|c|c|c|c|c|c|c|c|}
\hline Qualea parviflora & 37 & 32 & 3967,1 & 3448,9 & Ocotea corymbosa & 45 & 53 & 9006,8 & 9202,1 \\
\hline Aspidosperma subincanum & 36 & 37 & 2839,1 & 3139,5 & Rudgea viburnoides & 28 & 33 & 1008,3 & 1,331 \\
\hline Byrsonima crassa & 30 & 32 & 1986,1 & 2131,4 & Pera glabrata & 24 & 24 & 3290,8 & 3401,8 \\
\hline Aulomyrcia variabilis & 24 & 22 & 1229,3 & 1120,9 & Vatairea macrocarpa & 21 & 20 & 1920,3 & 1918,9 \\
\hline Leptolobium dasycarpum & 21 & 23 & 1024,2 & 1200,7 & Byrsonima laxiflora & 19 & 22 & 944,3 & 931,4 \\
\hline $\begin{array}{l}\text { Curatella americana } \\
\text { Chrysophyllum }\end{array}$ & 18 & 19 & 2665,7 & 2957,4 & Matayba guianensis & 16 & 14 & 1135,2 & 584,5 \\
\hline marginatum & 18 & 22 & 2232,3 & 2236,8 & Qualea parviflora & 15 & 13 & 2539,6 & 2736,3 \\
\hline Dalbergia miscolobium & 16 & 12 & 1256,1 & 1092,1 & Qualea multiflora & 15 & 13 & 534,9 & 518,3 \\
\hline Byrsonima coccolobifolia & 16 & 14 & 1566,5 & 1727,0 & Caryocar brasiliense & 15 & 7 & 3089,3 & 2048,2 \\
\hline Lithraea molleoides & 15 & 20 & 2421,2 & 2839,6 & $\begin{array}{l}\text { Dimorphandra mollis } \\
\text { Salvertia }\end{array}$ & 14 & 7 & 703,1 & 465,9 \\
\hline Astronium graveolens & 15 & 25 & 2673,9 & 3479,5 & convallariodora & 12 & 11 & 6291,9 & 5588,8 \\
\hline Diospyros hispida & 13 & 15 & 1074,8 & 1264,9 & Bowdichia virgilioides & 10 & 8 & 2512,9 & 2169,2 \\
\hline Sclerolobium aureum & 11 & 7 & 2004,7 & 1076,1 & Myrcia tomentosa & 9 & 5 & 443,6 & 363,6 \\
\hline Matayba guianensis & 9 & 10 & 1434,2 & 1639,7 & $\begin{array}{l}\text { Machaerium opacum } \\
\text { Stryphnodendron }\end{array}$ & 8 & 7 & 1010,3 & 852,3 \\
\hline Myrsine guianensis & 8 & 8 & 566,2 & 747,9 & polyphyllum & 7 & 5 & 185,6 & 135,1 \\
\hline Guazuma ulmifolia & 8 & 8 & 1012,7 & 1166,6 & Tapirira obtusa & 7 & 9 & 446,6 & 809,3 \\
\hline Xylopia aromatica & 7 & 16 & 441,6 & 1124,9 & Ocotea pulchella & 7 & 7 & 697,3 & 193,7 \\
\hline Tocoyena formosa & 7 & 8 & 363,8 & 495,1 & Maprounea guianensis & 7 & 8 & 910,2 & 1297,5 \\
\hline Erythroxylum deciduum & 7 & 7 & 500,3 & 543,4 & Heisteria ovata & 7 & 7 & 381,7 & 522,1 \\
\hline Dimorphandra mollis & 7 & 4 & 252,2 & 209,4 & Eriotheca gracilipes & 7 & 8 & 370,2 & 475,4 \\
\hline $\begin{array}{l}\text { Bowdichia virgilioides } \\
\text { Stryphnodendron }\end{array}$ & 7 & 7 & 1848,5 & 2066,4 & Brosimum gaudichaudii & 6 & 5 & 251,7 & 187,1 \\
\hline polyphyllum & 6 & 4 & 828,4 & 831,0 & Ficus sp. & 6 & 6 & 304,0 & 341,1 \\
\hline Senegalia polyphylla & 6 & 7 & 307,8 & 509,7 & Annona coriacea & 6 & 6 & 255,2 & 262,2 \\
\hline Salvertia convallariodora & 6 & 5 & 1427,9 & 1346,4 & Symplocos sp. & 6 & 1 & 309,3 & 81,5 \\
\hline Rudgea viburnoides & 6 & 5 & 258,5 & 186,5 & Styrax camporum & 6 & 6 & 169,8 & 256,7 \\
\hline Qualea multiflora & 6 & 6 & 513,4 & 567,3 & Platypodium elegans & 6 & 7 & 527,0 & 701,9 \\
\hline Myrcia rostrata & 6 & 7 & 387,1 & 483,1 & Copaifera langsdorffii & 6 & 6 & 2330,0 & 2949,3 \\
\hline Emmotum nitens & 6 & 6 & 818,7 & 1163,4 & Roupala montana & 5 & 4 & 383,8 & 356,9 \\
\hline Brosimum gaudichaudii & 5 & 5 & 119,4 & 161,5 & Callisthene major & 5 & 6 & 1431,2 & 1636,2 \\
\hline Aegiphila sellowiana & 5 & 1 & 513,7 & 81,5 & Dalbergia miscolobium & 4 & 3 & 1651,6 & 1182,3 \\
\hline Tabebuia aurea & 4 & 5 & 750,0 & 822,8 & Machaerium acutifolium & 4 & 3 & 113,6 & 125,9 \\
\hline Plathymenia reticulata & 4 & 5 & 398,0 & 546,1 & Schefflera macrocarpa & 4 & 2 & 181,4 & 134,7 \\
\hline Machaerium opacum & 4 & 4 & 684,2 & 726,9 & Qualea dichotoma & 4 & 6 & 169,7 & 326,6 \\
\hline Kielmeyera coriacea & 4 & 4 & 641,3 & 748,8 & Aulomyrcia variabilis & 3 & 3 & 221,0 & 217,4 \\
\hline Byrsonima laxiflora & 4 & 3 & 379,9 & 335,0 & Erythroxylum deciduum & 3 & 1 & 97,9 & 25,8 \\
\hline Agonandra brasiliensis & 4 & 4 & 712,1 & 802,3 & Annona crassiflora & 3 & 2 & 254,6 & 169,4 \\
\hline Plenckia populnea & 3 & 3 & 275,3 & 389,2 & Ouratea spectabilis & 3 & 3 & 751,7 & 759,6 \\
\hline Myrcia tomentosa & 3 & 3 & 115,4 & 118,7 & Ocotea spixiana & 3 & 2 & 128,0 & 131,8 \\
\hline Guapira noxia & 3 & 3 & 140,7 & 190,8 & Leptolobium elegans & 3 & 2 & 467,1 & 276,1 \\
\hline Ficus sp. & 3 & 3 & 3351,5 & 4343,7 & $\begin{array}{l}\text { Couepia grandiflora } \\
\text { Aspidosperma }\end{array}$ & 3 & 3 & 277,2 & 277,2 \\
\hline Erythroxylum daphnites & 3 & 5 & 188,8 & 288 & macrocarpon & 3 & 3 & 70,8 & 90,9 \\
\hline Annona coriacea & 3 & 2 & 330,2 & 84,4 & Myrsine umbellata & 2 & & 120,7 & \\
\hline Anadenanthera colubrina & 3 & 4 & 494,1 & 653,6 & Byrsonima crassa & 2 & 1 & 173,2 & 19,1 \\
\hline Vernonanthura ferruginea & 2 & 4 & 76,8 & 126,4 & Myrcia rostrata & 2 & 2 & 57,5 & 74,6 \\
\hline Tapirira guianensis & 2 & 4 & 224,7 & 502,2 & Plathymenia reticulata & 2 & 2 & 70,8 & 70,8 \\
\hline Sebastiana brasiliensis & 2 & 2 & 46,6 & 46,6 & $\begin{array}{l}\text { Guapira noxia } \\
\text { Heteropterys }\end{array}$ & 2 & 2 & 57,6 & 62,6 \\
\hline $\begin{array}{l}\text { Psidium pilosum } \\
\text { Pseudobombax }\end{array}$ & 2 & 2 & 61,5 & 61,5 & byrsonimifolia & 2 & 2 & 40,9 & 64,1 \\
\hline tomentosum & 2 & 1 & 3915,6 & 2381,7 & Hirtella gracilipes & 2 & 5 & 124,9 & 215,0 \\
\hline Pouteria rivicoa & 2 & 2 & 391,0 & 431,8 & $\begin{array}{l}\text { Hirtella americana } \\
\text { Handroanthus }\end{array}$ & 2 & 2 & 163,7 & 245,6 \\
\hline $\begin{array}{l}\text { Luehea divaricata } \\
\text { Handroanthus }\end{array}$ & 2 & 2 & 460,4 & 519,6 & serratifolius & 2 & 1 & 60,5 & 71,6 \\
\hline chrysotrichus & 2 & 2 & 177,3 & 300,0 & Guapira salicifolia & 2 & 2 & 136,0 & 206,8 \\
\hline Erythroxylum suberosum & 2 & 2 & 112,4 & 149,6 & Andira paniculata & 2 & 3 & 46,0 & 69,5 \\
\hline Cordia trichotoma & 2 & 2 & 80,9 & 80,9 & Terminalia argentea & 1 & 1 & 168,4 & 168,4 \\
\hline Connarus suberosus & 2 & 2 & 113,3 & 113,3 & Leptolobium & 1 & 1 & 19,1 & 25,8 \\
\hline
\end{tabular}




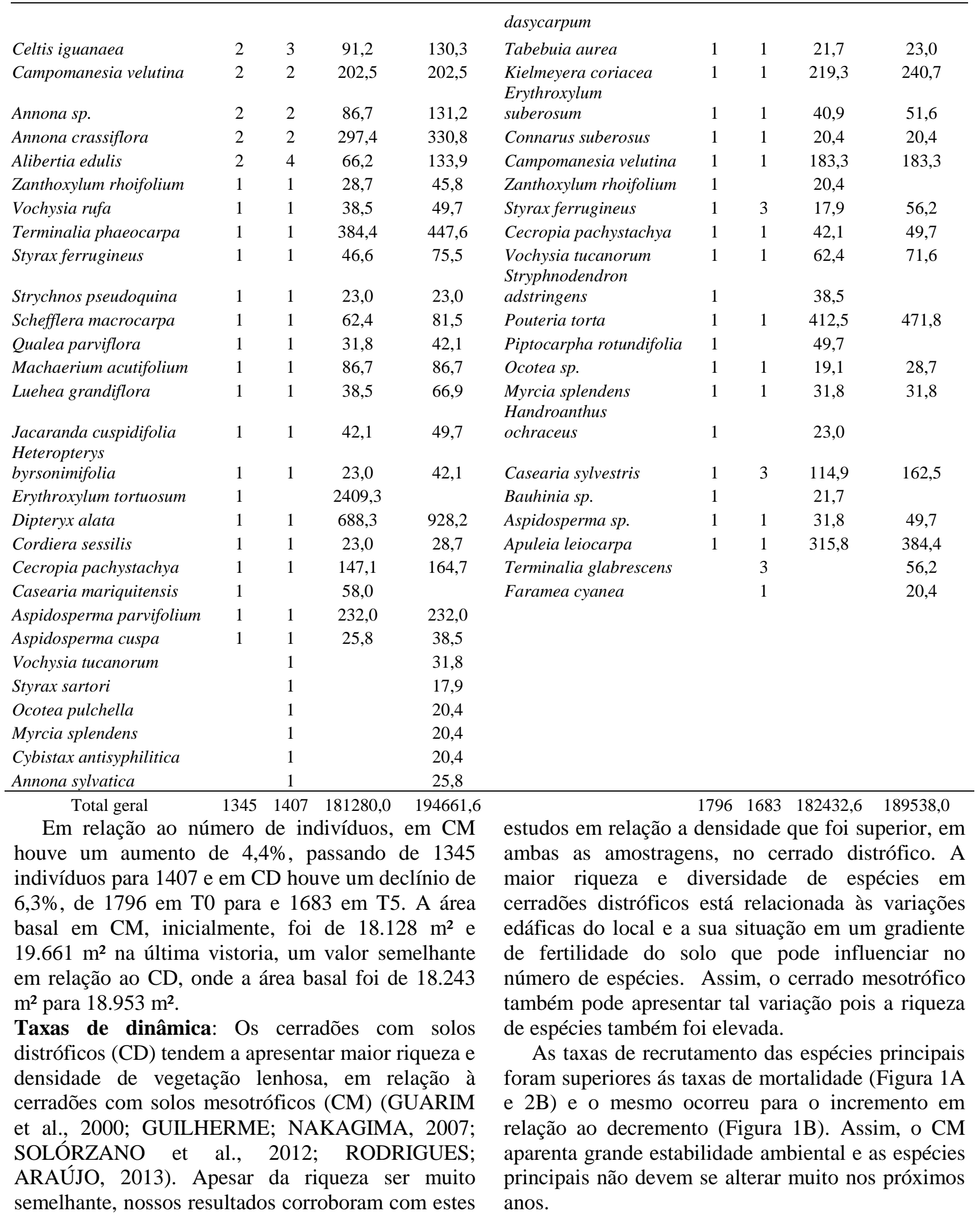



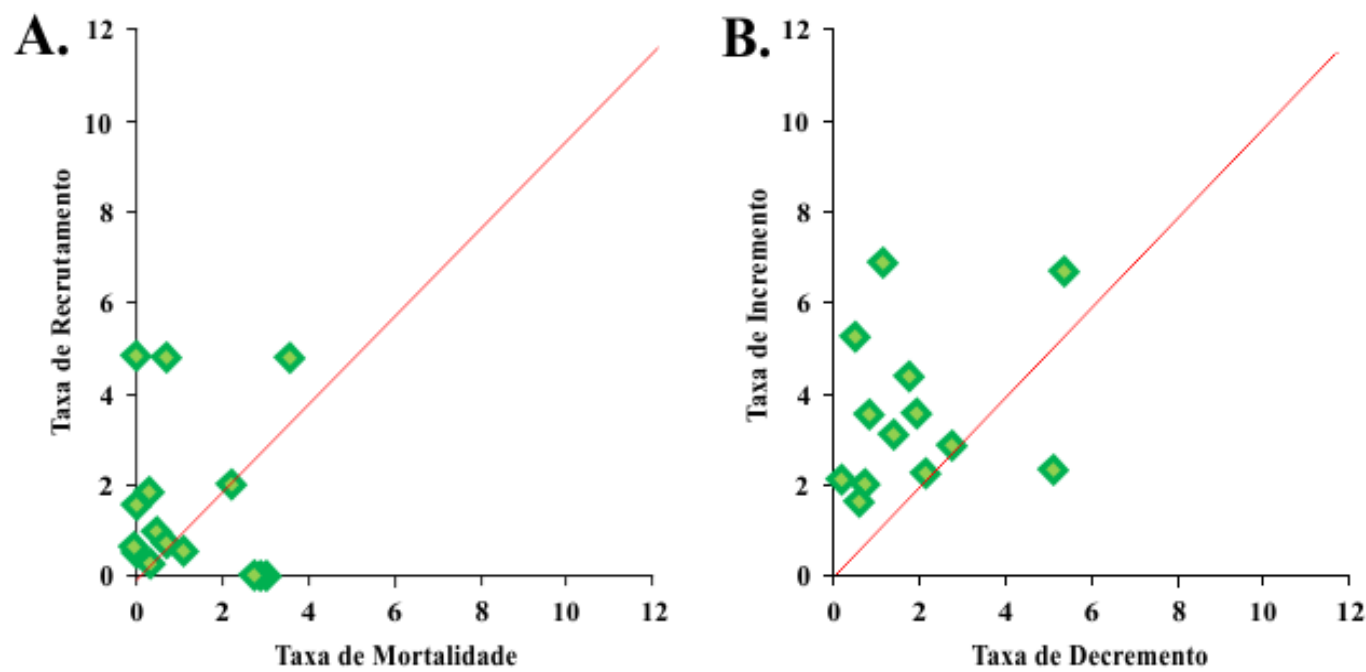

Figura 1 - Gráfico de dispersão gerado com os dados coletados no cerrado mesotrófico, a linha vermelha representando a divisão das taxas com resultado igual a 1. A. Taxa de Mortalidade x Taxa de Recrutamento. B. Taxa de Decremento x Taxa de Incremento.
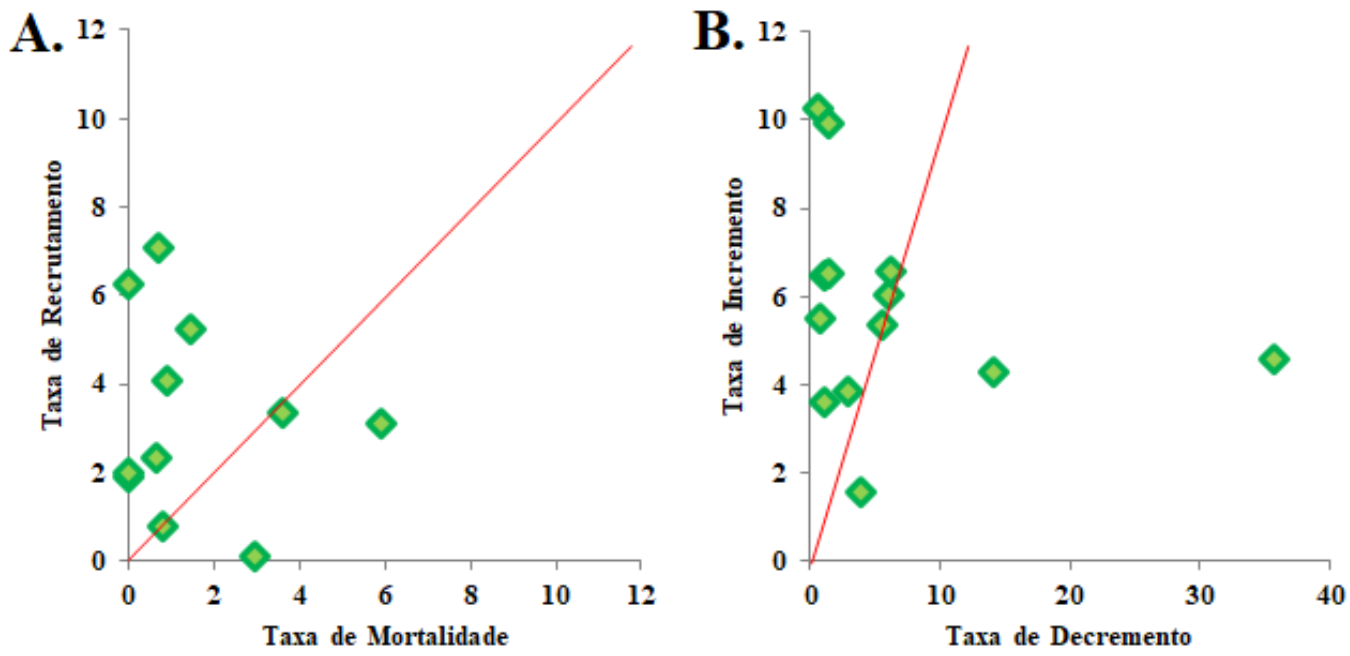

Figura 2 - Gráficos de dispersão gerado com os dados coletados no cerrado distrófico, a linha vermelha representando a divisão das taxas com resultado igual a 1. A. Taxa de Mortalidade x Taxa de Recrutamento. B. Taxa de Decremento x Taxa de Incremento.

Com relação ao número de indivíduos, o aumento, sempre constante, nas áreas basais, indica um provável processo de estabilidade da área. Porém as, apresentaram grande variação, enquanto Myracrodruon urundeuva, Virola sebifera, Cardiopethalum callophylum e Casearea grandiflora aumentaram um pouco suas densidades, Qualea grandiflora, Xylopia aromatica e Miconia albicans apresentaram alta mortalidade, sobretudo esta última que pode ser explicado pelo adensamento da floresta o qual ocasiona o sombreamento das espécies, como explicado por Franco (2005) que diferentes níveis de sombreamento podem definir a sucessão ou declínio de espécies na paisagem, e semelhante ao estudo anterior Werneck et al., (2000) atribuiu a diminuição do ciclo de vida dos indivíduos pela redução da disponibilidade luz. Mesmo que a área basal e a densidade tenham aumentado, as altas mortalidades dessas espécies indicam que a floresta está passando por uma fase de transição de espécies dominantes, de espécies mais típicas de cerrados para espécies mais típicas de ambientes florestais. A troca sucessional da área em CM pode ser apontada com baixa, sem grandes mudanças, podendo ser denominada como fase de Mata Secundária (KLEIN, 1980).

De modo distinto, CM registrou altas taxas de rotatividade. A Figura 2B demonstra que muitas espécies perderam em área basal e poucas espécies incrementaram. Estas mudanças nos indivíduos indicam que $\mathrm{CM}$ está em fase sucessional. Uma possível explicação para estas alternâncias, é a adaptabilidade das espécies locais. Entretanto, devese conhecer o histórico da área e realizar estudos referentes ao solo para verificar se essas alterações estão relacionadas ao meio edáfico. Os altos níveis de mortalidade podem ser relacionados a fatores naturais característicos em ambientes de cerradão, como idade, competição e supressão, condições climáticas e fogos (SANQUETTA, 1996) e não, 
necessariamente ao solo, mas como ambas as áreas estão na mesma região e não apresentam indícios de queima ou corte, é possível que ambas estejam passando por fases sucessionais distintas.

Além dos fatores naturais característicos de cerradão também há fatores que demonstram a estabilidade de uma floresta como o balanço entre mortalidade e recrutamento (MIGUEL et al., 2011) ou seja, não ocorrer um a diminuição de espécies e assim manter a densidade de indivíduos. Outro fator seria a área basal da floresta, o ganho e perda contribui para a redução de biomassa total da comunidade que é relacionado ao desbalanceamento entre as taxas de mortalidade e recrutamento (MEWS et al., 2011).

\section{CONCLUSÕES}

As espécies do cerradão distrófico apresentaram taxas de dinâmica mais estáveis enquanto que o cerradão mesotrófico apresentou maior variação entre as espécies principais. Apesar das comunidades aparentarem estabilidade, o CM parece estar em fase de sucessão e troca parcial das espécies principais. Analisar espécies individualmente auxilia na percepção das mudanças e indicam os progressos futuros da comunidade.

\section{REFERÊNCIAS}

COUTINHO, L. M. O conceito de Cerrado. Revista Brasileira de Botânica, v. 1, n. 1, p. 17-23, 1978.

FRANCO, A. Biodiversidade de forma e função: implicações ecofisiológicas das estratégias de utilização de água e luz em plantas lenhosas do Cerrado. In: SCARIOT, A.; SOUZA-SILVA, J. C.; FELFILI, J. M. (Orgs.) Cerrado: ecologia, biodiversidade e conservação. Brasília: Ministério do Meio Ambiente, 2005. p. 179-196 KLEIN, R. M. Ecologia da Flora e Vegetação do Vale do Itajaí. Sellowia, v. 32, p. 164-369, 1980.

KLINK, C. A., MACHADO, R. B. A conservação do Cerrado Brasileiro. Megadiversidade, v. 1, n.1, p. 148155, 2005.

HARIDASAN, M. Observations on soils, foliar nutrient concentration and floristic composition of cerrado sensu stricto and cerradão communities in central Brazil. In: FURLEY, P. A.; PROCTOR J.; RATTER, J.A. (eds.). Nature an Dynamics of Forest-Savanna Boundaries. London, Chapman \& Hall Publishing. 1992. P. 171-184.

HENRIQUES, R.P.B. Influência da história, solo e fogo na distribuição e dinâmica das fitofisionomias no bioma do Cerrado. In Cerrado: Ecologia, biodiversidade e conservação (A. Scariot, J. C. Souza-Silva; J.M. Felfili , eds.). Brasília: Ministério do Meio Ambiente. 2005. p.7392.
LOPES, A. S.; COX, F. R. Cerrado vegetation in Brazil: an edaphic gradient. Agronomy Journal, v. 69, n. 5, p. 828-831, 1977.

MARIMON JUNIOR, B. H.; HARIDASAN, M. Comparação da vegetação arbórea e características edáficas de um cerradão e um cerrado sensu stricto em áreas adjacentes sobre soo distrófico no leste de Mato Grosso, Brasil. Acta Botânica Brasílica, v. 19, n. 4, p. 913-926, 2005.

MIGUEL, A.; MARIMON, B. S.; OLIVEIRA, E. A.; MARACAHIPES, L.; MARIMON-JUNIOR, B. H. Dinâmica da comunidade lenhosa de uma floresta de galeria na transição Cerrado-Floresta Amazônica no leste de Mato Grosso, em um período de sete anos (1999 a 2006). Biota Neotropica, v. 11, n. 1, p. 53-63, 2011.

MIRANDA, S. C.; VASCONCELOS, W. A.; CEZARE, C. H. G.; MATA, C. R.; FONSEC, K. S. O cerradão de Goiás e suas relações florísticas e estruturais com ouras áreas no Brasil. Enciclopédia Biosfera, v. 14, n. 26, p. 1058-1069, 2017.

NAVES-BARBIERO, C. C.; FRANCO, A. C.; BUCCI, S. J.; GOLDSTEIN, G. Fluxo de seiva e condutância estomática de duas espécies lenhosas sempre-verdes no campo sujo e cerradão. Revista Brasileira de Fisiologia Vegetal, v. 12, n. 2, p. 119-134, 2000.

PRADO JÚNIOR, J. A.; LOPES, S. F.; VALE, V. S.; DIAS NETO, O. C.; SCHIAVINI, I. Comparação florística, estrutural e ecológica da vegetação arbórea das fitofisionomias de um remanescente urbano de cerrado. Bioscience Journal, v. 28, n. 3, p. 456-471, 2012.

RODRIGUES, R. F.; ARAÚJO, G. M. Estrutura da vegetação e características edáficas de um cerradão com solo distrófico e em solo mesotrófico no triângulo mineiro. Bioscience Journal, v. 29, n. 6, p. 2013-2029, 2013.

SANTOS, F. C.; NOVAIS, R. F.; NEVES, J. C. L.; FOLONI, J. M.; ALBUQUERQUE FILHO, M. R.; KER, J. C. Produtividade e aspectos nutricionais de plantas de soja cultivadas em solos de Cerrado com diferentes texturas. Revista Brasileira de Ciência do Solo, v. 32, n.5, p. 2015-2025, 2008

SHEIL, D.; BURSLEM, D.; ALDER, D. The interpretation and misinterpretation of mortality-rate reasures. Journal of Ecology, v. 83, p. 331-333, 1995.

SHEIL, D.; JENNINGS, S.; SAVILL, P. Long-term permanent plot observations of vegetation dynamics in Budongo, a Ugandan rain. Journal of Tropical Ecology, v. 16, p. 765-800, 2000.

SOUSA, D.M.G.; LOBATO, E., eds. Cerrado: correção do solo e adubação. 2.ed. Brasília, Embrapa, 2004. 416p. VELOSO, H.P.; RANGEL FILHO, A.L.R. \& LIMA, J.C.A. Classificação da vegetação brasileira, adaptada a um sistema universal. Rio de Janeiro, Fundação Instituto 
Brasileiro de Geografia e Estatística, Departamento de Recursos Naturais e Estudos Ambientais, 1991. 124p.

WALTER, B. M. T. Fitofisionomias do bioma Cerrado: síntese terminológica e relações florísticas. Brasília: UNB

- Departamento de Ecologia do Instituto de Ciências Biológicas, 2006. 373p. Tese de doutorado. 2006.
WERNECK, M. S.; FRANCESCHINELLI, E. V.; TAMEIRÃO-NETO, E. Mudanças na florística e estrutura de uma floresta decídua durante um período de quatro anos (1994-1998), na região do Triângulo Mineiro, MG. Revista Brasileira de Botânica, v. 23, n. 4, p. 401413, 2000. 